\title{
PESQUISA DE STAPHYLOCOCCUS AUREUS ENTEROTOXIGÊNICO NAS FOSSAS NASAIS DE MANIPULADORES DE ALIMENTOS EM HOSPITAIS, SÃO PAULO, 1976.
}

Sebastião Timo laria* Sirdéia M. P. Furlanetto * Maria Lúcia C. Campos*

\begin{tabular}{l|l|}
${ } }$ \\
\hline
\end{tabular}

IARIA, S. T. et al. Pesquisa de Staphylococcus aureus enterotoxigênico nas fossas nasais de manipuladores de alimentos em hospitais, São Paulo, 1976. Rev. Saúde públ., S. Paulo, 14:93-100, 1980.

RESUMO: Foi realizada a verificação da presença de Staphylococcus aureus enterotoxigênico, em manipuladores de alimentos, em cozinhas hospitalares. Foi colhido material da porção anterior das fossas nasais de 34 pessoas que trabalhavam em três hospitais da cidade de São Paulo. Dentre os individuos examinados, $12(35,3 \%)$ revelaram-se portadores de S. aureus e, destes $2(16,7 \%)$ foram positivos para cepas produtoras de enterotoxina esfafilocócica do tipo $C$. Das 12 cepas isoladas, 9 (75\%) foram fagotipáveis; das duas cepas enterotoxigênicas, uma foi náo fagotipável e a outra foi lisada por fagos do grapo III.

UNITERMOS: Staphylococcus aureus. Toxi-infecçóes alimentares estafilocócicas. Enterotoxinas.

\section{INTRODUÇAOO}

É sobejamente conhecido o envolvimento do Staphylococcus aureus em casos ou surtos de intoxicação ocasionados pela ingestão de alimentos contendo enterotoxinas produzidas por essa bactéria. Estes microorganismos vem causando intoxicações alimentares há séculos (Dack ', 1964). Segundo este autor, Vaughan em 1884 e Sternberg em 1885, trabalhando, independentemente, com queijos responsabilizados por casos de intoxicação alimentar, observaram a presença de estafilococos nestes alimentos. Em 1894, Denys assinalou pela primeira vez a presença de estafilococos piogênicos em carne de vaca morta por febre vitular, produto esse causador de um surto familiar de intoxicação alimentar. Em 1914, Barber relatou a presença de estafilococos em vaca com mastite, cujo leite havia provocado distúrbios gastrintestinais agudos em indivíduos que o ingeriram.

Mais recentemente, Brachman e col.4 (1973) referem que nos EUA em 1971 ocorreram 320 surtos de doenças de origem alimentar envolvendo 13.453 pessoas. Destes, 201 (63\%) atingindo 12.080 (90\%) individuos, foram atribuídos a infecçōes bacterianas. Do total de surtos, o S. aureus foi responsabilizado por $92(29 \%)$, com * Do Departamento de Microbiologia e Imunologia do Instituto de Ciências Biomédicas da
USP "Setor Saúde Pública" - Ar. Dr. Arnaldo, $715-01255-$ São Paulo, SP - Brasil. 
IARIA, S. T. et al. Pesquisa de Staphylococcus aureus enterotoxigênico nas fossas nasais de manipuladores de alimentos em hospitais, São Paulo, 1976. Rev. Saúde públ., S. Paulo, 14: 93-100, 1980.

$5.115(38 \%)$ pessoas atingidas. No Canadá, em $19 / 3$ o $S$. aureus foí responsável por 21 surtos que envolveram 600 pessoas, sendo este microrganismo o que figurou em primeiro lugar, devido ao maior número de surtos que provocou ${ }^{14}$.

Por outro lado, é bem conhecida a partrcipação de portadores de $S$. aureus na cadeia epidemiológica da intoxicação alimentar estafilocócica. Entretanto, segundo Williams $^{41}$ (1963), os estudos sobre portadores de $S$. aureus só puderam ser realizados após a difusāo do uso da prova da coagulase nos EUA por Chapman em 1934 e na Inglaterra por Cruickshank em 1937.

A colonização nasal do $S$. aureus no homem é descrita freqüentemente e uma proporção grande de pessoas "normais" mantém este microrganismo em suas fossas nasais, constituindo-se em seu maior reservatório. Ludlan 19 (1953) estudando crianças lactentes com sete semanas de vida, verificou que a taxa de portadores nasais de S. aureus era de $59,3 \%$ e Castello e Maggia ${ }^{6}$ (1951) em pesquisa semelhante constataram a taxa de $80 \%$ em recém-nascidos.

A proporção de adultos "normais", não associados a hospitais, portadores dessa bactéria, está compreendida entre 30 e $50 \%$, sendo que esta taxa aumenta para 60 a $80 \%$ em pessoal hospitalar (Williams, 1963) ${ }^{41}$. Moss e col. ${ }^{23}$ (1948) examinando material de fossas nasais colhido por meio de zaragatoa, encontrou estafilococos em $32 \%$ das pessoas estudadas enquanto que em material obtido do vestíbulo nasal essa proporção era de $76 \%$. O estudo de Jacobs e col.17 (1961) revelou resultados similares, porém Stratford e col.3i (1960) encontraram positividade de $51 \%$ de portadores. Investigações realizadas nos EUA, Inglaterra e Australia por Williams 4: (1946), Ridley ${ }^{33}$ (1969) e Martin e Whitehead 21 (1948) mostraram uma freqüência de portadores nasais de $44 \%, 42 \%$ e $40 \%$, respectivamente, em adultos "normais".
Possati 27 (1955), Findlay e Abrahams 13 (1946) e Rountree ${ }^{30}$ (1956) observaram em pessoas assintomáticas taxas de positividades para S. aureus de $38 \%, 30 \%$ e $20 \%$, respectivamente.

Nos últimos anos, tem sido realizado um número considerável de investigações sobre a epidemiologia das infecções estafilocócicas cruzadas no ambiente hospitalar 2,12,28,29,32. Estas pesquisas revelam que as taxas de portadores de $S$. aureus em pessoal hospitalar assim como em pacientes são altas 20,26. Mostram ainda que o reservatório principal desse agente infeccioso são as fossas nasais de portadores, a partir dos quais os pacientes se infectam com maior frequiência. Entretanto, Zelante ${ }^{44}$ (1974) citando vários autores, refere que a área bucal, possivelmente, é tão importante quanto a nasal como reservatório de S. aureus.

Pelas pesquisas anteriormente referidas pode-se depreender que a frequiencia de portadores de $S$. aureus é relativamente alta e, tratando-se de indivíduos que manipulam alimentos, podem constituir-se em elementos importantes na cadeia epidemiológica da intoxicação alimentar estafilocócica, desde que estejam infectados com $S$. aureus produtores de enterotoxina.

Assim, com base no exposto e dada a ausência de estudos dessa natureza com relação a indivíduos que trabalham no preparo de alimentos em nosso meio, planejou-se a realização da presente pesquisa, que tem por objetivos verificar a presença de $S$. aureus nas fossas nasais de manipuladores de alimentos em cozinhas de hospitais, pesquisar a capacidade produtora de enterotoxina estafilocócica das cepas isoladas, assim como verificar a que fagotipos pertencem tais cepas.

\section{MATERIAL E METODOS}

Foi colhido material das fossas nasais de 34 pessoas, sem sinais clínicos aparentes de infeç̧ão estafilocócica, que trabalha- 
IARIA, S. T. et al. Pesquisa de Staphylococcus aureus enterotoxigênico nas fossas nasais de manipuladores de alimentos em hospitais, São Paulo, 1976. Rev. Saúde públ, S. Paulo, 14: $93-100,1980$.

vam em cozinhas de três hospitais do município de São Paulo, manipulando diretamente alimentos. O material foi obtido do vestíbulo nasal por meio de zaragatoa, sendo esta posteriormente colocada em tubo estéril, contendo cerca de $0,5 \mathrm{ml}$ de caldo simples e levada imediatamente ao laboratório para exame.

Isolamento e identificação de Staphylococcus aureus - Para o isolamento desta bactéria, o material da zaragatoa era semeado na superfície de ágar simples adicionado de $10 \%$ de sangue desfibrinado de coelho, em placa de Petri, a qual era incubada a $37^{\circ} \mathrm{C}$ por $24-48$ horas. A seguir, em cada caso, realizava-se o isolamento de uma colônia com as características das de estafilococos, a qual era semeada em tubo de ágar simples inclinado. Após 24 horas de incubação a $37^{\circ} \mathrm{C}$ eram preparados esfregaços corados pelo método de Gram, para a verificação da morfologia das cepas isoladas. A seguir, as cepas revelando-se como cocos Gram-positivos dispostos em cachos eram submetidas às provas de verificação da produção de catalase (Baird-Parker ${ }^{1}, 1966$ ), da fermentação-oxidação da glicose, segundo a técnica preconizada pelo Subcomitê Internacional de Taxonomia de Staphylococcus e Micrococcus 38 (1965), da produção de DNase (Di Salvo ${ }^{10}, 1958$ ) e de coagulase livre, de acordo com a técnica usada por Zelante ${ }^{44}$ (1974).

Fagotipagem - A caracterização dos fagótipos das cepas isoladas de $S$. aureus foi realizada no Laboratório de Estrepto$\operatorname{cocos}$ e Estafilococos, Seção de Fagotipagem, do Departamento de Parasitologia, Microbiologia e Imunologia da Faculdade de Medicina de Ribeirão Preto - USP, sendo a metodologia adotada a proposta por Blair e Williams ${ }^{3}$ (1961). Foram utilizados nas provas, 23 fagos do conjunto básico internacional (Solé-Vernin ${ }^{36}, 1976$ ),
7 experimentais (86, 88, 89, 90, 92, D11 e HK2) e dois extras (187 e 42D). Todas as cepas isoladas de $S$. aureus foram submetidas à ação de 1xRTD ("Routine Test Dose") e, quando não reagiam a esta dose, procedia-se à prova com 100xRTD (Solé-Vernin ${ }^{36}$ 1976).

Pesquisa da produção de enterotoxina pelas cepas isoladas - $\mathrm{Na}$ produçāo de enterotoxina pelas cepas isoladas, foi empregada a técnica de cultura de $S$. aureus em saco de celofane, preconizada por Donnelly e col.11 (1967), com base no estudo realizado por Simkovicova e Gilbert 35 (1971). Na prova de dupla imunodifusão em lâmina (Siles-Villarroel ${ }^{34}, 1972$ ), utilizamos, como padrões, as enterotoxinas estafilocócicas A, B, C, D e E e as respectivas anti-enterotoxinas.*

\section{RESULTADOS}

A Tabela 1 mostra a distribuição dos manipuladores de alimentos, segundo 0 hospital em que trabalhavam, a positividade para $S$. aureus e o tipo de enterotoxina elaborada pelas cepas isoladas. Dos 34 manipuladores, $12(35,3 \%)$ revelaram-se portadores de $S$. aureus no vestibulo nasal, sendo $3(17,6 \%)$ pertencentes aos 17 examinados do hospital " $X$ ", $7(58,3 \%)$ aos 12 do "Y" e 2 (40\%) aos 5 do " $Z$ ". Um manipulador do hospital " $Y$ " e um do " $Z$ " revelaram-se portadores de $S$. aureus produtor de enterotoxina.

Na Tabela 2 estão distribuidas as cepas de $S$. aureus isoladas, segundo a fagotipagem. Nove $(75 \%)$ das 12 cepas estudadas foram lisadas por um ou mais dos fagos utilizados e $3(25 \%)$ revelaram-se não tipáveis. Das duas cepas positivas para a produção de enterotoxina $C$, uma foi fagotipável e a outra não.

\footnotetext{
* Preparadas e gentilmente fornecidas pelo Professor Merllin S. Bergdoll do "Food Research Institute" da Universidade de Wisconsin, EUA.
} 
IARIA, S. T. et al. Pesquisa de Staphylococcus aureus enterotoxigênico nas fossas nasais de manipuladores de alimentos em hospitais, São Paulo, 1976. Rev. Saúde públ., S. Pálo. 14: $93-100,1980$.

\section{T A B E L A 1}

Distribuição de manipuladores de alimentos de très hospitais, segundo a positividade para $S$. aureus no vestíbulo nasal e a produção de enterotoxina pelas cepas isoladas, São Paulo, 1976.

\begin{tabular}{|c|c|c|c|c|c|}
\hline \multirow{3}{*}{ Hopital } & \multirow{3}{*}{$\begin{array}{l}\text { Manipuladores } \\
\text { examinados }\end{array}$} & \multicolumn{4}{|c|}{ Positivos para $\mathbf{S}$. aureus } \\
\hline & & \multirow[b]{2}{*}{ No } & \multirow[b]{2}{*}{$\%$} & \multicolumn{2}{|c|}{ Produtor de enterotoxina } \\
\hline & & & & $\mathrm{N}$ & Tipo \\
\hline " $\mathrm{X} "$ & 17 & 3 & 17,6 & - & - \\
\hline "Y" & 12 & 7 & 58.3 & 1 & $\mathrm{C}$ \\
\hline$" z "$ & 5 & 2 & 40,0 & 1 & $\mathrm{C}$ \\
\hline Total & 34 & 12 & 35.3 & 2 & \\
\hline
\end{tabular}

T A B E L A 2

Distribuição das 12 cepas de $S$. aureus isoladas do vestíbulo nasal dos manipuladores de alimentos, segundo a fagotipagem e a produção de enterotoxina. São Paulo, 1976.

\begin{tabular}{|c|c|c|c|c|c|}
\hline \multicolumn{2}{|c|}{ Fagotipagem } & \multicolumn{4}{|c|}{ Cepas de $s$ aureus } \\
\hline \multirow[b]{2}{*}{ Grupos } & \multirow[b]{2}{*}{ Tipos } & \multirow[b]{2}{*}{$N^{\prime \prime}$} & \multirow[b]{2}{*}{$\%$} & \multicolumn{2}{|c|}{$\begin{array}{c}\text { Produtoras de } \\
\text { enterotoxina }\end{array}$} \\
\hline & & & & No & Tipo \\
\hline $\mathrm{I} / \mathrm{NC}$ & $29 / 52 / 95$ & 1 & 8.3 & - & - \\
\hline $\mathrm{I} / \mathrm{NC}$ & $52 / 80 / 81$ & 1 & 8.8 & - & - \\
\hline II & 71 & 1 & 8.3 & - & - \\
\hline II & 55 & 1 & 8,8 & - & - \\
\hline III & 53 & 1 & 8.3 & - & - \\
\hline III & $42 \mathrm{E}$ & 1 & 8.3 & - & - \\
\hline III & $47 / 53 / 54 / 75 / 77 / 83 \mathrm{~A} / 84 / 85$ & 1 & 8,3 & 1 & $\mathrm{C}$ \\
\hline Exper. & D11 & 1 & 8,3 & - & - \\
\hline Exper. & 88 & 1 & 8,3 & - & - \\
\hline- & Não tipáveis & 8 & 25,0 & 1 & $\mathrm{C}$ \\
\hline
\end{tabular}

NC - Não classificados

\section{DISCUSSÃO}

Analisando-se a Tabela 1 pode-se verificar que a taxa de positividade para $S$. aureus, observada para as fossas nasais dos manipuladores de alimentos examinados, foi relativamente alta $(35,3 \%)$. Entre- tanto, nota-se uma variação dessa taxa quando se consideram os individuos estudados, de acordo com os hospitais em que trabalhavam. Assim, os manipuladores de alimentos do hospital " $\mathrm{X}$ " revelaram positividade de $17,6 \%$, ou seja, de metade da verificada no hospital " $Z$ " e de um terço da 
IARIA. S. T. et al. Pesquisa de Staphylococcus aureus enterotoxigênico nas fossas nasais de manipuladores de alimentos em hospitais. São Paulo, 1976. Rer. Saúde públ., S. Paulo, 14: $93-100,1980$.

observada no "Y". A menor taxa de positivos para $S$. aureus observada nos manipuladores de alimentos do hospital " $\mathrm{X}$ " foi, provavelmente, devida ao fato de que nele é realizado periodicamente o controle de portadores, segundo informaçōes que nos foram fornecidas.

Considerando-se ainda os resultados obtidos para os três grupos de manipuladores, separadamente, pode-se depreender que os mesmos concordam com os encontrados por vários pesquisadores. Assim Martin e Whitehead 21 (1948) verificaram uma taxa de portadores de $40 \%$, Possati $2-$ (1955) de $38 \%$, Rountree ${ }^{30}$ (1956) de $20 \%$ e Torres e col.39 (1974) de 18,3\%. Em pessoas normais doadoras de sangue em hospitais, Rountree e Rheuben ${ }^{31}$ (1956) observaram que a proporção de portadores era de 50\%. Millian e col.2* (1960) estudaram a presença de $S$. aureus em manipuladores de alimentos e constataram que $38 \%$ deles eram portadores desta bactéria nas fossas nasais.

Na presente investigação, considerandose os 34 manipuladores de alimentos examinados, pode-se verificar que a positividade para S. aureus observada concorda com a encontrada por Millian e col.22 (1960), porém difere das constatadas por Torres e col. ${ }^{39}$ (1974) e Del Campo ${ }^{9}$ (1957) os os quais verificaram taxas de portadores de $94,2 \%$ e $18,3 \%$, respectivamente.

Ainda na Tabela 1 pode-se constatar que das 12 cepas de $S$. aureus isoladas, apenas $2(16,7 \%)$ revelaram-se produtoras de enterotoxina. É de ressaltar-se, no entanto, que foram isoladas de manipuladores de alimentos que trabalhavam na cozinha de hospitais diferentes. A este respeito, deve ser salientado que os portadores de $S$. aureus na indústria de produtos alimenticios ou em locais onde se preparam alimentos, são importantes com relação à epidemiologia da intoxicação alimentar estafilocócica. Quando um manipulador é portador de $S$. aureus, na maioria das vezes contamina o alimento através das mãos ou de goticulas oro-nasais e, havendo condições favoráveis à multiplicação dessa bactéria, os seus consumidores podem correr risco de contraírem intoxicação alimentar. Assim, neste estudo, embora tenhan sido revelados somente dois portadores de $S$. aureus enterotoxigênico, o fato é importante do ponto de vista epidemiológico, relativamente à possibilidade da ocorrência de casos de intoxicação alimentar estafilocócica nos hospitais respectivos.

$\mathrm{Na}$ Tabela 2 verifica-se que ambas as cepas enterotoxigênicas mostraram-se produtoras de enterotoxina estafilocócica do tipo C. Casman e col.j (1967) e Hajek e Marsalek 1.i (1973) verificaram que as cepas de $S$. aureus por eles isoladas de pessoas e de animais eram produtoras de enterotoxinas dos tipos D e E. Muller e col::4 (1973) relatam o encontro de $74 \%$ de cepas produtoras de enterotoxinas dos tipos A, $\mathrm{B}$ e $\mathrm{C}$ entre as enterotoxigênicas isoladas de casos clinicos de infecção estafilocócica c das fossas nasais de portadores de $S$. aureus. Wieneke ${ }^{10}$ (197t), estudando cepas de $S$. aureus isoladas de pessoas com sinais de infecção e de alimentos, verificou que a frequiência de cepas produtoras de enterotoxina do tipo $D$ era maior que dos tipos A, B, C e E. Em nosso meio, Delazari e Leitão * (1976) constataram, em macarrão, predominância de cepas produtoras de enterotoxina do tipo A, com pequena ocorrência da do tipo C. Por outro lado, laria ${ }^{4 i}$ (1978) verificou, em cepas enterotoxigênicas isoladas de doces cremosos, maior percentual de positividade para as produtoras de enterotoxina do tipo $C$.

De acordo com Jay is (1973), as tentativas para estabelecer relação entre a capacidade enterotoxigênica e a fagotipagem do $S$. auteuts têm fracassado, porém parece que a maior parte das cepas produtoras de intoxicação alimentar têm se revelado sensiveis a fagos do grupo 111 do 
IARIA, S. T. et al. Pesquisa de Staphylococcus aureus enterotoxigênico nas fossas nasais de manipuladores de alimentos em hospitais, São Paulo, 1976. Rev. Saúde públ., S. Paulo, 14: 93-100, 1980.

conjunto básico internacional. Por outro lado, segundo esse autor e Munch-Petersen 25 (1961), a maioria das cepas isoladas de alimentos e de pessoas com sinais de infeç̧ão, também são lisadas por fagos do grupo III. Entretanto, Simkovicova e Gilbert ${ }^{35}$ (1971) e Wieneke 40 (1974) verificaram que comumente são isoladas cepas de $S$. aureus associadas a surtos de intoxicação alimentar, sensiveis a fagos dos grupos 11 ll ou $1 /$ III. Em nosso meio, Iaria 16 (1978) verificou que de 9 cepas de $S$. aureus enterotoxigênicas, isoladas de amostras de doces cremosos, nenhuma delas foi lisada pelos fagos do conjunto básico internacional, assim como pelos demais fagos utilizados. Na presente pesquisa (Tabela 2), das duas cepas enterotoxigênicas isoladas, uma foi sensivel a fagos do grupo III e a outra não foi fagotipável.

\section{CONCLUSOES}

Os resultados obtidos permitem-nos apresentar as seguintes conclusões:

1. Em 34 individuos que manipulam alimentos em três hospitais, a proporção de portadores nasais de Staphylococcus aureus foi de $35,3 \%$, taxa esta que pode ser considerada alta.

2. Dois $(16,7 \%)$ dos individuos examinados e que trabalhavam em dois hospitais revelaram-se positivos para $S$. aureus produtor de enterotoxina do tipo $\mathrm{C}$.

3. Das 12 cepas de $S$. aureus isoladas de 12 individuos positivos, 9 (75\%) foram fagotipáveis. Das duas cepas produtoras de enterotoxina do tipo $\mathrm{C}$, uma foi lisada por fagos do grupo III e a outra não foi fagotipável.

IARIA, S. T. et al. [Staphylococcus aureus in the anterior portion of nasal fossae of hospital food handlers, São Paulo, Brazil, 1976.l Rev. Saúde púb., S. Paulo, 14:93-100, 1980.

ABSTRACT: The presence of enterotoxic $S$. aureus in the nasal fossae of hospital food handlers was investigated. Material was collected from the anterior portion of fossae of 34 food handlers in the kitchens of three hospitals in the city of S. Paulo, Brazil. Of these 34 persons, $12(35.3 \%)$ were positive for $\mathrm{S}$. aureus, of these same 12 , two $(16.7 \%)$ showed positive for type C staphylococcal enterotoxin producing strains. Of the 12 strains isolated, nine $(75 \%)$ could be phagetyped; of the enterotoxic strains, one was lysed by Group III phage; the other was non-typeable. rotoxins.

UNITERMS: Staphylococcus aureus. Staphylococcal food poisoning. Ente-

\section{REFERENCIAS BIBLIOGRÁFICAS}

1. BAIRD-PARKER, A. C. Methods for classifying staphylococci and micrococci. In: Gibbs, B. M. Skinner, F. A. Identification methods for microbiologists. London, Academic Press, 1966. p. $59-64$.
2. BALDWIN, J. N. et al. Staphylococcal infections in newborn infants. III. Colonization of newborn infants by Staphylococcus pyogenes A. W. A. Amer, J. Dis. Child., 94:107-12, 1957. 
IARIA, S. T. et al. Pesquisa de Staphylococcus aureus enterotoxigênico nas fossas nasais de manipuladores de alimentos em hospitais, São Paulo, 1976. Rev. Saúde públ., S. Paulo, 14: $93-100,1980$.

3. BLAIR, J. E. \& WILLIAMS, R. E. O. Phage-typing of staphylococci. Bull. Wld Hith Org., 24:771-84, 1961.

4. BRACHMAN, $P$. et al. Food poisoning in USA. In: Hobbs, C. B. \& Christian. J. H. B. The microbiological safety of food. New York, Academic Press, 1973. p. $143-51$.

5. CASMAN, E. P. et al. Indentification of a fourth staphylococcal enterotoxin $D$. J. Bact., 94:1875-82, 1967.

6. CAStello, I. \& MAGGia, O. Ricerche sulla presenza nella mucosa del neonato nei primigiorni di vita di stafilococchi con particolare referimento ala loro sensibilita o meno alla penicillina. Gior. Batt. Immun., 43:120-3. 1951.

7. DACK, G. M. Food poisoning. 3rd ed. Chicago, University of Chicago, 1964. p. 109-58,

8. DELAZARI, I. \& LEITAO, M. F, F, Staphylococcus aureus enterotoxigênico em macarrão. Col. Inst. Tecnol. Alim., $7: 485-97,1976$.

9. DEL CAMPO, A. Diffusione e caractteristiche degli stafilocochi nella popolazione sana. Nuovi Ann. Ig., 8:385-94, 1957.

10. DI SALVO, G. W. Desoxyrribonuclease and coagulase activity of micrococci. Med. Techn. Bull., 9:191-6, 1958.

11. DONNELLY, C. B. et al. Sorological identification of enterotoxigenic staphylococci from cheese. Appl. Microbiol., 15:1382-7, 1967.

12. FEKETY, F. R. et al. Control of outbreak of staphylococcal infection among mothers and infants in suburban hospital. Amer. $J$. publ. Hlth, 43:298310, 1958.

13. FINDLAY, G. M. \& ABRAHAMS, C. Staphylococcal incidence in the nose and on the skin of africans and europeans in West Africa. J. roy. Army med. Cps, 87 :272-4. 1946.

14. FOOD-BORNE DISEASE IN CANADA: Annual summary 1973. (Health Protection Branch, Health and Welfare) Ottawa, 1976.
15. HAJEK, V. \& MARSALEK, E. The occurrence of enterotoxigenic Staphylococcus aureus strains in hosts of different animal species. $\mathrm{Zbl}$. Bkt. Reihe A, 223:63-8, 1973. Apud Abstr. Hyg., 48:463, 1973.

16. IARIA, S. T. Staphylococcus aureus em doces vendidos em padarias $e$ confei. tarias do municipio de são Paulo. Produção de enterotoxina estafilocócica $e$ fagotipagem a partir das cepas isoladas (1975/1976). São Paulo, 1978. [Tese de Livre-Docência - Instituto de Ciências Biomédicas da USP]

17. JACOBS, S. I. et al. Nasal abnormality and the carrier rate of Staphylococcus aureus. J. clin. Path., 14:519-21, 1961.

18. JAY, M. J. Microbiologia moderna de los alimentos. Zaragoza, Ed. Acribia, 1973. p. 190-206.

19. LUDLAM, G. M. Incidence and penicillin gensitivity of Staphylococcus aureus in the nose in infants and their mothers. J. Hyg., $51: 64-74,1953$.

20. MAC FARLAN, A. M. Incidence of pathogenic staphylococci in the nose. Brit. med. J., 2:939-41, 1958.

21. MARTIN, T. D. M. \& WHITEHEAD, M. E. J. Carriage of penicillin resistant Staphylococcus pyogenes in healthy adults. Brit. med. J., 1:173-5, 1948.

22. MILliaN, S. J. et al. Studies on incidence of coagulase positive staphylococci in a normal unconfined population. Amer. $J$, publ. Hlth, 50:791-8, 1960.

23. MOSS, B. et al. Nose and skin carriage of Staphylococcus aureus in patients receiving penicillin. Lancet, 1:320-5, 1948.

24. MULLER, $H$, et al. Fecal carrier rate and enterotoxigenicity of Staphylococcus aureus in man. $2 b l$. Bkt. Reihe A., 2:180-96, 1973. Apud Excerpta med. Sect. 17, 22:438-9, 1974.

25. MUNCH-PETERSEN, E. Staphylococcal carriage in man. Bull. Wla Flth Org., $24: 761-9,1961$.

26. PACKALEN, T. \& BERGOVIST, S. Staphylococci in throat and nose and staphylolysin titer. Acta med. scand., 127: 291-312, 1947. 
IARIA, S. T, et al. Pesquisa de Staphylococcus aureus enterotoxigènico nas fossas nasais de manipuladores de alimentos em hospitais, São Paulo, 1976. Rev. Saúde públ., S. Paulo, 14: $93-100,1980$.

27. POSSATI, F, Ricerca di portatori di stipiti di stafilococco dotati di caratteri biochimici di patogenicita in um grupo di 100 saggetti. Riv. ital. Igiene, 15:504-9, 1965 .

28. RAVENHOLT, R. T. \& RAVENHOLT. D. H. Staphylococcal infections in the hospital and community. Amer. J. publ. Hith, $43: 277-87,1958$.

29. RAVENHOLT, $R$, $T$. et al. The epidemiology and prevention of nursey derived staphylococcal disease. New Engl. $J$. Med., 257:789-91, 1957.

30. ROUNTREE, P. M. Staphylococci harboured by people in Western Highlands of New Guinea. Lancet, 1:719-20, 1956.

31. ROUNTREe, P. M. \& RHEUBEN, J. Penicillin resistent staphylococci in the general population. Med. J. Aust., 1: $399-402,1956$.

32. ROUNTREE, $P$. $M$. et al. Control of staphylococcal infection of newborn by treatment of nasal carriers in the staff. Med. J. Aust., 1:530-6, 1956.

33. RIDLEY, M. Perineal carriage of Staphylococcus aureus. Brit. Med. J., 1: $270-3,1959$.

34. SILES-VILLARROEL, M. Contribuicão ao estudo de verienos de serpentes do gênero Bothrops (B. jararaca, B, alternatus, B. insularis. B. jaracussu B. athrox e B, cotiara). São Paulo, 1972. [Tese de Doutoramento - Instituto de Ciências Biomédicas da USP]

35. SIMKOVICOVA, M. \& GILBERT, R. J. Serological detection of enterotoxin from food-poisoning strains of Staphylococcus aureus. $J$. med. Microbiol., 4:19-30, 1971.
36. SOLÉ-VERNIN, C. Fagotipagem de Staphylococcus aureus. Rev, bras. Ort., 11: $31-4,1976$.

37. STRATFORD, B. et al. Treatment of the nasal carrier of Staphylococcus aureus with framycetin and other antibacterials. Lancet, $2: 1225-7,1960$.

38. SUBCOMMITTEE on Taxonomy of Staphylococei and Micrococci. Minutes of First Meeting (5th-6th October. 1964). Int. Bull. bact. Nomencl., 15:107-8, 1965.

39. TORRES, R. A. et al. Estudio de los caracteres biologicos de estafilococos aislados de manipuladores de alimentos. Rev. Sanid. Hig. públ., 48:113960, 1974 .

40. WIENEKE, A. A. Enterotoxin production by strains of Staphylococcus aureus isolated from foods human beings. $J$. Hyg., London, 73:255-62, 1974.

41. WILlIAMS, R. E. O. Healtly carriage of Staphylococcus aureus, Bact. Rev., 27: 56-71, 1963.

42. WILliams, R. E. O. Skin an nose carriage of bacteriophage types of Staphylococcus aureus. J. Path. Bact., 58: 259-68, 1946.

43. WILSON, G. S. \& MILES, A. A. Topley and Wilson's principles of bacteriology and immunity. 5th ed. London. Edward Arnold Ltd., 1966. p. 746-79.

44. ZELANTE, F. Contribuicão para o estudo de Staphylococcus isolados de canais radiculares. São Paulo, 1974. [Tese de Livre-Docência - Instituto de Ciências Biomédicas da USP]

Recebido para publicação em 19/09/1979 Aprovado para publicação em 30/10/1979 